\title{
Validation experiment for large-sample prompt-gamma neutron activation analysis
}

\author{
M. Blaauw, ${ }^{1 *}$ I. H. Degenaar, ${ }^{1}$ C. Yonezawa, ${ }^{2}$ H. Matsue, ${ }^{2}$ J. J. M. de Goeij ${ }^{1}$ \\ ${ }^{1}$ Reactor Institute Delft, Faculty of Applied Sciences, University of Technology Delft, Mekelweg 15, 2629JB Delft, The Netherlands \\ ${ }^{2}$ Japan Atomic Energy Research Institute, Tokai, Ibaraki 319-1195, Japan
}

(Received April 13, 2006)

\begin{abstract}
A method is proposed for the implementation of large-sample prompt-gamma neutron activation analysis (LS-PGNAA). The method was tested with four different sample materials at the thermal PGNAA facility at JAERI, Japan. The macroscopic scattering cross section $\left(\Sigma_{s}\right)$ and absorption cross section $\left(\Sigma_{a}\right)$ of the samples were determined by monitoring the neutron flux in four positions just outside the sample container. With the $\Sigma_{s}$ and $\Sigma_{a}$ determined, the spatial neutron density distribution $[n(r)]$ inside the sample material was derived. Taking $n(r)$ and the gamma-ray selfabsorption into account simultaneously, the effective geometric gamma-ray detection efficiency for large samples as a function of gamma-ray energy was calculated. Taking silicon as test element, the concentrations found agreed to within $7 \%$ with the known concentrations in the four sample materials examined, both when using relative standardization and with absolute standardization.
\end{abstract}

\section{Introduction}

The methodology proposed for LS-PGNAA and tested in this paper consists of several steps:

In the first step, the values for the scattering and absorption neutron cross sections $\Sigma_{s}$ and $\Sigma_{a}$ of the sample material are derived experimentally. For that purpose the relations derived and tested in two preceding papers are used. In the first of these, a method was presented to determine the macroscopic scattering and absorption cross sections $\left(\Sigma_{s}\right.$ and $\left.\Sigma_{a}\right)$ of samples using the probability of capture of neutrons in copper foils $(P)$ located at four positions outside the sample. ${ }^{1}$ In the second, ${ }^{2}$ it was concluded that the effective mass $\left(M_{e}\right)$ of the atoms in the sample was actually not influencing the accuracy of the determination of mass fractions in LS-PGNAA.

In the second step, the neutron-density distribution, $n(r)$, inside the sample material is simulated using the $\Sigma_{s}$ and $\Sigma_{a}$ values derived in the first step. In step three, the effective geometric counting efficiency of the detector for the sample is calculated, using the simulated $n(r)$, taking the gamma-attenuation in the sample material into account. Basically, MoENs' effective solid-angle method $^{3}$ is applied for each volume voxel, the resulting correction factor multiplied with the local neutron density, and the volume integral taken. The result is a photon-energy dependent correction factor that takes both neutron self-shielding and gamma self-absorption into account.

The element mass fractions are calculated by relative standardization, i.e., by using a calibration sample, and by absolute standardization, i.e., the silicon mass fractions were calculated from various nuclear and other constants in combination with the measured neutron flux.
The validation experiment performed at the JAERI, Japan, to validate the methodology outlined above, involved four sample materials, selected to have different neutron transport properties and different gamma attenuations. The macroscopic cross sections and the gamma-attenuation coefficient were different for the four samples, but were all within the restrictions derived in References 1 and 2, that is, the total neutron cross section $\Sigma_{t}$ is smaller than $0.6 \mathrm{~cm}^{-1}$ and the ratio $\Sigma_{s} / \Sigma_{t}$ is larger than 0.1 .

\section{Experimental}

\section{Sample preparation}

One sample material was chosen to have a composition with $\Sigma_{s}$ and $\Sigma_{a}$ considered typical for samples to be analyzed in the future $\left(\mathrm{SiO}_{2}\right)$, one sample material to have a large $\Sigma_{a}$ (a mixture of $\mathrm{SiO}_{2}$ and $0.103 \mathrm{~g} / \mathrm{g} \mathrm{Li}_{2} \mathrm{SO}_{4}$ ) and two samples to have a large $\Sigma_{s}$ ( $\mathrm{SiC}$, and $\mathrm{SiO}_{2}$ mixed with $0.409 \mathrm{~g} / \mathrm{g}$ starch). Both mixtures were homogenized in a turbulator for about 24 hours each. The other sample materials were used as received.

All constituents were obtained from Aldrich. $\mathrm{SiO}_{2}$ had a 325 mesh and a purity of $99.6 \%$. SiC had a 400 mesh and a purity of about $100 \%$. The starch was soluble Amylopecti. The $\mathrm{Li}_{2} \mathrm{SO}_{4}$ had a purity of $99 \%$. The isotope ratio ${ }^{6} \mathrm{Li} /{ }^{7} \mathrm{Li}$ was determined with ICP-MS and was equal to the natural isotopic ratio.

The materials were placed in cylindrical 1-liter PTFE (polytetrafluoroethene) bottles with an outer diameter of $10 \mathrm{~cm}$, an outer height of $16 \mathrm{~cm}$ and a wall thickness of $0.35 \mathrm{~cm}$. The sample heights inside the bottles were initially equal for the different sample materials, but

\footnotetext{
* E-mail: blaauw@iri.tudelft.nl
} 
changed between the filling and the experiment, due to compaction of the material. The material characteristics, theoretical macroscopic scattering cross sections, as well as the sample masses and heights at the time of experiment, are shown in Fig. 1.

The tabulated values of the density $\rho$ were calculated from the mass of the sample material, the element composition and the sample dimensions (Table 1). The $\Sigma_{t}$ and $\Sigma_{s} / \Sigma_{t}$ ratio shown were calculated using the density $(\rho)$ and the microscopic cross sections $\left(\sigma_{a}\right.$ and $\sigma_{s}$ ) taken from JEF 2.2. ${ }^{4}$ The $\Sigma_{t}$ of these sample materials was smaller than $0.6 \mathrm{~cm}^{-1}$ and the $\Sigma_{s} / \Sigma_{t}$ ratio was larger than 0.1 , as required by the findings in Reference 1.

For calibration purposes a small sample, with dimension $1.0 \times 1.0 \times 0.1 \mathrm{~cm}^{3}$, was packed in $25 \mu \mathrm{m}$ FEP (tetrafluoropropene) film. This small sample consisted of a mixture of $213.50 \mathrm{mg} \mathrm{SiO}_{2}$ and $12.48 \mathrm{mg} \mathrm{NH}_{4} \mathrm{Cl}$.

Four copper foils, of a purity of $99.98 \%$ and purchased from Aldrich, were taped to the outside of each of the sample containers with $\operatorname{Scotch}^{\circledR}$ tape. The foils had a height and width of $1.0 \mathrm{~cm}$ each and a thickness of $0.025 \mathrm{~cm}$ and were weighted before use. The positions of the copper foils are shown in Fig. 1. One foil was situated between the sample and the neutron source.

The backside of the foils at the angles of $45^{\circ}$ and $135^{\circ}$ was covered with Aldrich cadmium foil with a purity of $99.9998 \%$ and dimensions of

\section{Top view \\ $(\mathbf{z}, \mathbf{x})$}

$2.5 \times 2.5 \times 0.15 \mathrm{~cm}^{3}$. This was done to protect these copper foils from activation due to neutrons which did not come directly from the sample.

\section{Facilities}

The irradiation facility used was the thermal PGNAA facility of the JRR-3M reactor at JAERI, Japan. ${ }^{5}$ Even though this beam is guided, it has a virtually purely Maxwellian-shaped energy distribution corresponding to a temperature of about $200 \mathrm{~K}$, and is collimated to a square cross section of $2.0 \times 2.0 \mathrm{~cm}^{2}$.

The activity of the copper flux monitor foils was measured through the $511 \mathrm{keV}$ annihilation photons, with an Ortec GMX type 30\% efficiency, closed-end coaxial type high-purity Ge detector at a distance of 0 or $5 \mathrm{~cm}$ from the end cap, depending on the activity of the copper foil. This detector was located in a lead castle far away from the PGNAA system, and exhibited low background count rates. The PGNAA spectra of the samples were recorded with another Ortec GMX type $30 \%$ efficiency Ge detector with a FWHM of $2.4 \mathrm{keV}$ at $1333 \mathrm{keV}$.

The efficiency curve determined previously by RAMAN et al. $^{6}$ for the PGNAA detector was used, and related to the efficiency curves of the other detector used for the flux monitors by using a certified ${ }^{152} \mathrm{Eu}$ source.

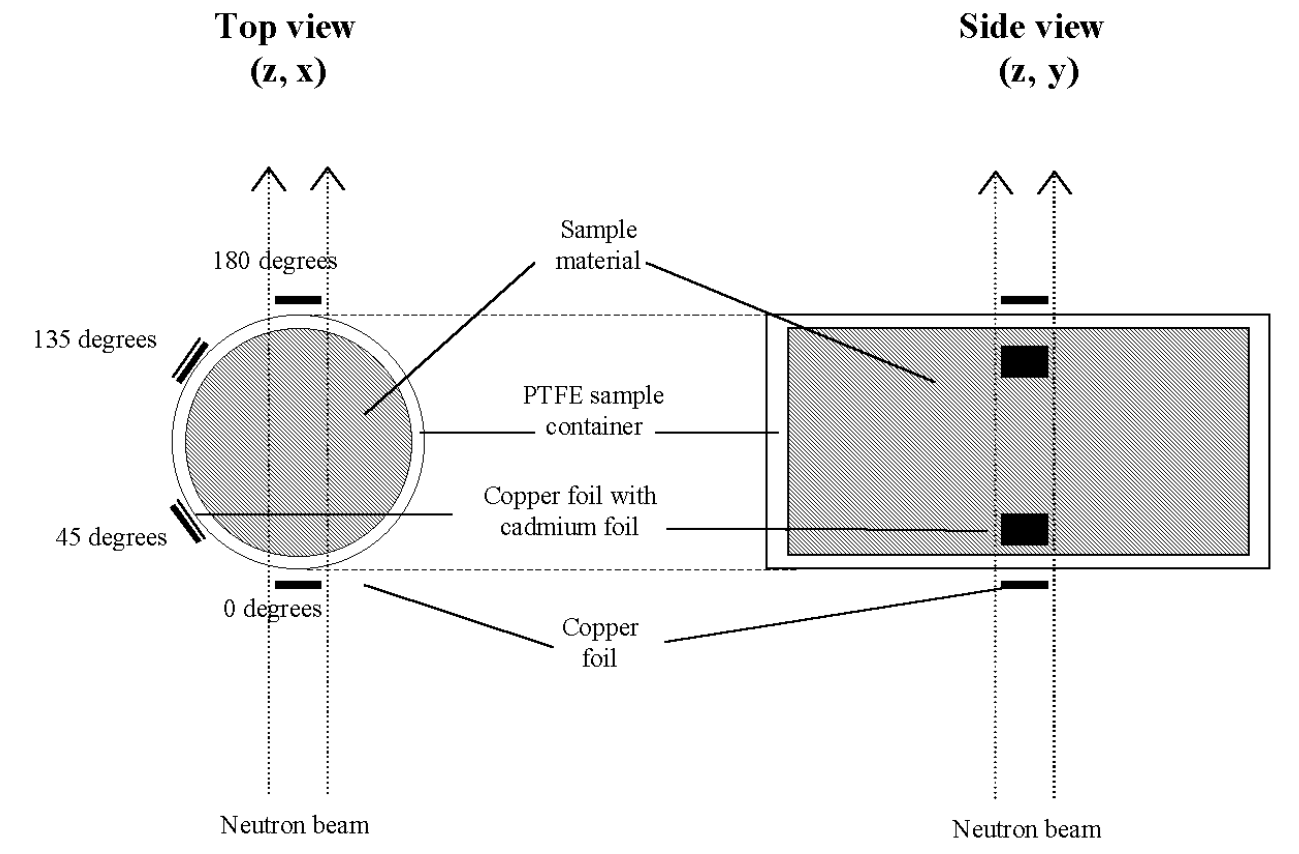

Fig. 1. Setup geometry of the simulation: the PTFE bottle being irradiated with an (undisturbed) neutron beam and the position of the copper flux monitors. Neutrons come in along the $Z$-axis, the cylindrical sample is aligned along the $Y$-axis, and the detector is placed at the positive $X$-axis 
Table 1. Characteristics of the samples and materials used in the experiment

\begin{tabular}{lccccc}
\hline \multicolumn{1}{c}{ Sample type } & $\rho, \mathrm{kg} \cdot \mathrm{m}^{-3}$ & $\Sigma_{t}, \mathrm{~cm}^{-1}$ & $\Sigma_{\mathrm{s}} / \Sigma_{t}$ & Sample mass, kg & Sample height, cm \\
\hline $\mathrm{SiO}_{2}$ & 650 & $6.67 \cdot 10^{-2}$ & 0.985 & 0.65 & 13.9 \\
$\mathrm{SiC}$ & 1470 & $1.51 \cdot 10^{-1}$ & 0.974 & 1.48 & 13.9 \\
$\mathrm{SiO}_{2}$ and starch & 700 & $5.05 \cdot 10^{-1}$ & 0.992 & 0.70 & 13.9 \\
$\mathrm{SiO}_{2}$ and $\mathrm{Li}_{2} \mathrm{SO}_{4}$ & 840 & $2.25 \cdot 10^{-1}$ & 0.693 & 0.65 & 10.4 \\
\hline
\end{tabular}

$\Sigma_{t}$ and $\Sigma_{s}$ are the total and scattering neutron cross sections, respectively, calculated from literature data and known sample compositions.

\section{Procedure}

The four samples and the blank sample, i.e., an empty sample container, were irradiated in two steps with the beam perpendicular to the longitudinal symmetry axis, $Z$-axis, of the sample container.

During a first irradiation of 6 up to 8 hours the PGNAA spectrum of each sample material was recorded. During the second irradiation of about 16 hours no PGNAA spectrum was recorded, but the sample was irradiated together with the four copper foils positioned outside the sample container.

A separate experiment was performed to ensure that the center of mass of the sample container was positioned in the middle of the beam. In this experiment, a $4 \times 4 \mathrm{~cm}$ copper foil was attached to the empty sample container, cut into sections afterwards, and gamma-ray spectra measured, in order to obtain a map of neutron density as a function of position in the end.

\section{Data handling}

The observed $511 \mathrm{keV}$ emission rates were converted to neutron densities during irradiation for all copper foil measurements using the data in Table 2. (The $1346 \mathrm{keV}$ line was observed in all copper spectra, as opposed to the sodium lines, but the $511 \mathrm{keV}$ yielded the better counting statistics.) The values for $\Sigma_{s}$ and $\Sigma_{a}$ of the samples were determined using the relations found in Reference 1 and the neutron densities observed in the copper foils outside the sample. The values of $\Sigma_{s}$ and $\Sigma_{a}$ determined were used as input parameters for the calculation of $n(r)$ inside the sample material by our inhouse Monte Carlo program BUDA. ${ }^{7}$ The experimental set-up described in the input file of BUDA was the real set-up of the PGNAA facility at JAERI. A grid of $10 \times 10$ voxels over the cross section of the cylinder and 20 voxels in the length direction of the sample was chosen for this calculation. BUDA was also used to determine $f$ for all sample materials with an imprecision smaller than $0.02 \%$.
Table 2. Nuclear data applied in the calculation of the silicon masses in the absolute standardization method

\begin{tabular}{lcc}
\hline & ${ }^{28} \mathrm{Si}(\mathrm{n}, \gamma)^{29} \mathrm{Si}$ & ${ }^{63} \mathrm{Cu}(\mathrm{n}, \gamma){ }^{64} \mathrm{Cu}$ \\
\hline$M, \mathrm{~g} \cdot \mathrm{mol}^{-1}$ & 27.980 & 62.930 \\
$\sigma_{a}, \mathrm{~b}$ & 0.1718 & 3.79 \\
$\theta$ & 0.923 & 0.692 \\
$I_{\gamma}(1273.4 \mathrm{keV})$ & 0.160 & - \\
$I_{\gamma}(2093.1 \mathrm{keV})$ & 0.185 & - \\
$I_{\gamma}(3539.0 \mathrm{keV})$ & 0.664 & - \\
$I_{\gamma}(511.0 \mathrm{keV})$ & - & 0.379 \\
$\lambda, \mathrm{s}^{-1}$ & - & $1.52 \cdot 10^{-5}$ \\
\hline
\end{tabular}

For each voxel, the method of MoENs ${ }^{3}$ was then applied to get the full energy detection efficiency as a function of gamma-ray energy, relative to the efficiency for a point source at positioned at the center of the neutron beam in the sample. Using the BUDA results, the neutron density in the voxels was also known relative to a point sample in the beam. By taking the volume integral of the product of these two, an efficiency curve was obtained taking both neutron selfshielding and gamma self-absorption into account allowed for interpretation of the PGNAA spectrum as if it were the spectrum of a point sample.

The peak areas of three neutron-capture lines of ${ }^{28} \mathrm{Si}$ $(1273.4 \mathrm{keV}, \quad 2093.1 \mathrm{keV}$ and $3539.0 \mathrm{keV})$ in all PGNAA spectra were determined using the peak fitting method described by BlAAUw. ${ }^{8}$ This method searches for peak locations and determines the peak areas in a spectrum.

The full-energy neutron-capture peaks of other elements did not interfere with energy peaks of silicon in the sample material, except for the neutron-capture peak of ${ }^{35} \mathrm{Cl}$ in the calibration sample at $2091.8 \mathrm{keV}$, which interfered with the $2093.1 \mathrm{keV}$ neutron-capture peak of silicon. Using the efficiency curve and tabulated ${ }^{9}$ prompt-gamma yields, this interference was corrected for.

Pile-up corrections were performed using the method described by LINDSTROM and FLEMING, ${ }^{10}$ using the MCA-registered dead time and the actual dead time measured with a pulser in the later experiments. 
The silicon masses of the large samples were calculated from the gamma-ray spectra in three ways. First, it was calculated via the relative standardization method using the calibration sample, without the gamma and neutron shielding corrections (In this approach, the observed amount of silicon was divided by the mass of sample in the beam to get a concentration). Second, it was calculated with the corrections and relative standardization. Third, it was calculated via absolute standardization in which the silicon masses were calculated directly from the PGNAA spectrum without a calibration sample, but using the neutron fluxes observed with the copper foils exclusively.

To use absolute standardization, nuclear parameters were used as shown in Table 2.

\section{Results}

The profile of the neutron-beam flux $(\varphi)$ as determined with the $4 \times 4 \mathrm{~cm}$ square copper foil is shown in Table 3. The imprecision due to counting statistics of all pieces of copper foil was smaller than $2 \%$. The imprecision in the cutting of the copper foil was smaller than $0.5 \mathrm{~mm}$. A correction for this cutting error was carried out by taking the masses of the copper foil sections into account in the calculation of $\varphi$. All other sources of imprecision are considered negligible. In this table it can be seen that $\varphi$ is not entirely uniform over the beam area and that the CM of the sample container was placed virtually exactly in the middle of the neutron beam. Still, the simulations with BUDA were carried out as if the neutron beam was uniform.

The results of the determination of $\Sigma_{s}$ and $\Sigma_{a}$ are displayed in Table 4. The $f$ of the small calibration sample was calculated to be $1.0037 \pm 0.02 \%$.
The results of the relative standardization obtained without any correction for neutron self-shielding and gamma-attenuation are displayed in Table 5, with corrections in Table 7, and with absolute standardization and corrections in Table 6.

\section{Discussion}

The silicon concentrations measured in this experiment agree reasonably well with the known values. But when the experimental results shown in Table 4 are compared to the $\Sigma_{s}$ and the $\Sigma_{s} / \Sigma_{t}$ ratios calculated from literature values in Table 1 , it can be seen that the values do not agree to within the stated uncertainties. This may be the result of the fact that the relations between the copper foil neutron densities and the $\Sigma_{s}$ and the $\Sigma_{s} / \Sigma_{t}$ found in Reference 1 were based on a simulated circular homogeneous beam with a diameter of $2.54 \mathrm{~cm}$ and a sample container with sample material of $20 \mathrm{~cm}$ height, while in the real experiments a square sample beam was used with a width of $2 \mathrm{~cm}$ and the sample heights ranged from 10.4 to $13.9 \mathrm{~cm}$.

Table 3. Neutron beam intensity profile (in $10^{11} \mathrm{~m}^{-2} \cdot \mathrm{s}^{-1}$ ) at the position where the neutron beam enters the sample container, in the plane perpendicular to the neutron beam. The outer 12 copper foils had dimensions $1 \times 1 \mathrm{~cm}^{2}$, the middle 16 copper foils had dimensions $0.5 \times 0.5 \mathrm{~cm}^{2}$

\begin{tabular}{|l|l|l|l|l|l|}
\hline 0.04 & \multicolumn{2}{|c|}{0.29} & \multicolumn{2}{c|}{0.28} & 0.08 \\
\hline \multirow{2}{*}{0.07} & 3.06 & 5.57 & 6.98 & 6.41 & \multirow{2}{*}{1.21} \\
\cline { 2 - 5 } & 3.38 & 6.66 & 7.29 & 6.31 & \\
\hline \multirow{2}{*}{0.06} & 2.76 & 5.27 & 6.60 & 6.08 & \multirow{2}{*}{0.97} \\
\cline { 2 - 5 } 0.05 & 2.20 & 5.41 & 5.67 & 5.29 & \multirow{2}{*}{0.26} \\
\cline { 2 - 4 } & \multicolumn{3}{|c}{0.60} & \multicolumn{3}{c|}{0.91} & 0.26 \\
\hline
\end{tabular}

The thick-bordered area indicates the position of the $1 \times 1 \mathrm{~cm}^{2}$ calibration sample. The imprecision in the counting statistics was smaller than $2 \%$.

Table 4. The results for $\Sigma_{s}$ and $\Sigma_{t}$ determined for the large samples

\begin{tabular}{lllll}
\hline & \multicolumn{1}{c}{$\mathrm{SiO}_{2}$} & \multicolumn{1}{c}{$\mathrm{SiC}$} & \multicolumn{1}{c}{$\mathrm{SiO}_{2}+$ starch } & \multicolumn{1}{c}{$\mathrm{SiO}_{2}+\mathrm{Li}_{2} \mathrm{SO}_{4}$} \\
\hline$\Sigma_{t}, \mathrm{~cm}^{-1}$ & $0.0923(0.4)$ & $0.16(0.3)$ & $0.647(0.2)$ & $0.203(0.2)$ \\
$\Sigma_{S} / \Sigma_{t}$ & $0.910(0.5)$ & $0.97(0.4)$ & $0.95(0.2)$ & $0.49(0.4)$ \\
$f_{L}$ & $0.0351(0.02)$ & $0.0366(0.02)$ & $0.0314(0.02)$ & $0.0292(0.02)$ \\
\hline
\end{tabular}

In the last row the self-shielding factor $f_{t}$ is displayed that was calculated with BUDA using the determined $\Sigma_{s}$ and $\Sigma_{t}$ as input parameters. The 1 s.d. imprecision in percents is given between brackets.

Table 5. Mass of silicon in the different samples present and calculated without corrections for neutron self-shielding and gamma-attenuation and using the relative standardization method

\begin{tabular}{lcccc}
\hline & $\mathrm{SiO}_{2}$ & $\mathrm{SiC}$ & $\mathrm{SiO}_{2}+$ starch & $\mathrm{SiO}_{2}+\mathrm{Li}_{2} \mathrm{SO}_{4}$ \\
\hline 'real' $m_{\mathrm{Si}}$ & 0.30 & 1.03 & 0.19 & 0.27 \\
$m_{1272}$ & 0.25 & 0.61 & 0.12 & 0.17 \\
$m_{2093}$ & 0.29 & 0.74 & 0.14 & 0.19 \\
$m_{3539}$ & 0.31 & 0.83 & 0.15 & 0.21 \\
\hline
\end{tabular}


Table 6. Silicon mass in the large samples determined with the absolute standardization LS-PGNAA methodology

\begin{tabular}{lllll}
\hline & \multicolumn{1}{c}{$\mathrm{SiO}_{2}$} & \multicolumn{1}{c}{$\mathrm{SiC}$} & \multicolumn{1}{c}{$\mathrm{SiO}_{2}+$ starch } & \multicolumn{1}{c}{$\mathrm{SiO}_{2}+\mathrm{Li}_{2} \mathrm{SO}_{4}$} \\
\hline 'real' $m_{\mathrm{Si}}$ & 0.304 & 1.028 & 0.194 & 0.271 \\
$m_{1272}$ & $0.312(0.3)$ & $0.925(0.8)$ & $0.181(0.7)$ & $0.259(0.7)$ \\
$m_{2093}$ & $0.327(0.5)$ & $0.966(0.7)$ & $0.183(0.6)$ & $0.264(0.6)$ \\
$m_{3539}$ & $0.332(0.6)$ & $0.995(0.8)$ & $0.192(0.6)$ & $0.266(0.5)$ \\
$m_{a}$ & $0.324(10)$ & $0.962(12)$ & $0.185(10)$ & $0.263(4.4)$ \\
$m_{a} / m$ & 1.07 & 0.94 & 0.95 & 0.97 \\
\hline
\end{tabular}

In the first row the known silicon masses, $m$, are given. In the next three rows the masses determined per energy peak are given together with the 1 s.d. relative uncertainty in percents due to counting statistics between brackets. In the fifth row the average, $m_{a}$, is given together with the relative $95 \%$ confidence levels of the Student's $t$-distribution in percents. In the last row the ratios between the masses $\left(m_{a} / m\right)$ are given.

Table 7. Silicon mass in the large samples determined with the relative standardization LS-PGNAA methodology

\begin{tabular}{lllll}
\hline & \multicolumn{1}{c}{$\mathrm{SiO}_{2}$} & \multicolumn{1}{c}{$\mathrm{SiC}$} & \multicolumn{1}{c}{$\mathrm{SiO}_{2}+$ starch } & \multicolumn{1}{c}{$\mathrm{SiO}_{2}+\mathrm{Li}_{2} \mathrm{SO}_{4}$} \\
\hline 'real' $m_{\mathrm{Si}}$ & 0.304 & 1.03 & 0.194 & 0.271 \\
$m_{1272}$ & $0.321(0.3)$ & $0.950(0.8)$ & $0.186(0.7)$ & $0.266(0.7)$ \\
$m_{2093}$ & $0.335(0.5)$ & $0.988(0.7)$ & $0.187(0.6)$ & $0.270(0.6)$ \\
$m_{3539}$ & $0.332(0.6)$ & $0.993(0.8)$ & $0.191(0.6)$ & $0.266(0.5)$ \\
$m_{a}$ & $0.329(7.1)$ & $0.977(7.7)$ & $0.188(4.5)$ & $0.267(2.7)$ \\
$m_{a} / m$ & 1.08 & 0.95 & 0.97 & 0.99 \\
\hline
\end{tabular}

In the first row the known silicon mass, $m$, is given. In the next three rows the masses determined per energy peak are given together with the 1 s.d. relative uncertainty in percents due to counting statistics between brackets. In the fifth row the average, $m_{a}$, is given together with the relative $95 \%$ confidence levels of the Student's $t$-distribution in percents. In the last row the ratios between the masses $\left(m_{a} / m\right)$ are given.

Nevertheless, since the inaccuracy of the final concentration results is better than the targeted $10 \%$, the relations of Reference 1 can be used to determine $\Sigma_{s}$ and $\Sigma_{a}$ for the experimental setup at the PGNAA facility at JAERI. It should be noted that, in the second step of the procedure described in the introduction, the $n(r)$ inside the sample material was simulated with the square beam and the correct height of the sample material in the sample container height. The sample height in itself is not a likely cause of the discrepancy, because very few neutron diffuse to the uppermost and lowermost layers of sample. The low neutron densities observed in the copper flux monitors at 45 and 135 degrees, positioned at a few $\mathrm{cm}$ distance from the beam, demonstrate this point. The neutron beam shape and homogeneity are the more likely causes of discrepancy.

Inhomogeneous samples, being the more interesting ones in LS-PGNAA, would result in less accuracy. The effects of inhomogeneity can be suppressed, however, by sample rotation and translation during the measurement. Trace element distributions of inhomogeneous trace elements in a homogeneous matrix could even be determined that way.

The neutron shadow of the copper foil at 0 degrees has no effects: The transmission of $0.025 \mathrm{~cm}$ of $\mathrm{Cu}$ is very close to unity and moreover, the foils were present in the Monte Carlo calculations as well as in the experiments, resulting in cancellation of any effects.

\section{Conclusions}

The silicon mass fractions determined agreed to within $8 \%$ with the true silicon mass fractions in four different sample materials using the proposed LSPGNAA methodology with either relative standardization, as shown in Table 7 or to within $7 \%$ using the absolute standardization methodology, as shown in Table 6.

It is expected that also other elements than silicon can be determined with an inaccuracy better than $10 \%$ in sample materials within the range of sample characteristics tested, since the limiting factor is not the element of interest, but the sample matrix characteristics, $\Sigma_{s}, \Sigma_{a}$ and $M_{e f f}$.

The method for the determination of $\Sigma_{s}$ and $\Sigma_{a}$ of the sample material could be used even though the neutron beam and the sample container and material had slightly different dimensions, as long as the correct values were used in the simulation of the $n(r)$ in the sample material. 


\section{References}

1. I. H. Degenaar, M. BlaAuw, J. J. M. De GoeiJ, J. Radioanal. Nucl. Chem., 271 (2007) 765.

2. M. BlaAuw, I. H. DegenaAR, J. J. M. De GoeiJ, J. Radioanal. Nucl. Chem., 271 (2007) 761.

3. L. Moens, J. DE DOnder, X. Lin, F. DE CORTe, A. De Wispelaere, A. Simonits, J. Hoste, Nucl. Instr. Meth., 187 (1981) 451.

4. OECD, Table of Simple Integral Neutron Cross-section Data from JEF-2.2, ENDF/B-VI, JENDL-3.2, BROND-2 and CENDL-2, Paris, France, 1994.

5. C. YoneZAwA, Anal. Sci., 9 (1993) 185.
6. S. Raman, C. Yonezawa, H. Matsue, H. Iimura, N. SHINOHARA, Nucl. Instr. Meth., A454 (200) 389.

7. M. BLAAUW, BUDA, A Program for Incoherent Neutron Scattering Monte Carlo Computations, Version 8, Reactor Institute Delft, Delft, The Netherlands, 2000.

8. M. BlaAuw, The Holistic Analysis of Gamma-ray Spectra in Instrumental Neutron Activation Analysis, PhD Thesis, Technical University Delft, 1993.

9. R. S. ReEdy, S. C. Frankle, Evaluated Database for Prompt Gamma from Radiative Capture of Thermal Neutrons by Elements from Hydrogen Through Zinc, cd-rom, 2002.

10. R. M. Lindstrom, R. F. Fleming, 6 (1995) 20. 